\title{
THERMAL TREATMENT OF STARCH SLURRY IN COUETTE-TAYLOR FLOW APPARATUS
}

\author{
Robert Hubacz $^{* 1}$, Hayato Masuda ${ }^{2,3}$, Takafumi Horie ${ }^{3}$, Naoto Ohmura ${ }^{3}$ \\ ${ }^{1}$ Warsaw University of Technology, Faculty of Chemical and Process Engineering, ul. Waryńskiego \\ 1, 00-645 Warszawa, Poland \\ ${ }^{2}$ University of Shizuoka, School of Food and Nutritional Science, 52-1 Yada, Suruga, Shizuoka 422- \\ 8526, Japan
}

${ }^{3}$ Kobe University, Graduate School of Engineering, Department of Chemical Science and Engineering, 1-1 Rokkodai, Nada, Kobe 657-8501, Japan

\begin{abstract}
In this paper, thermal processing of starch slurry in a Couette-Taylor flow (CTF) apparatus was investigated. Gelatinized starch dispersion, after treatment in the CTF apparatus, was characterized using such parameters like starch granule diameters (or average diameter), starch granule swelling degree (quantifying the amount of water absorbed by starch granules) and concentration of dissolved starch. These parameters were affected mostly by the process temperature, although the impact of the axial flow or rotor rotation on them was also observed. Moreover, the analysis of results showed a relatively good correlation between these parameters, as well as, between those parameter and apparent viscosity of gelatinized starch dispersion. Meanwhile, the increase in the value of the apparent viscosity and in shear-tinning behaviour of dispersion was associated with the progress of starch processing in the CTF apparatus. Finally, the CTF apparatuses of different geometries were compared using numerical simulation of the process. The results of the simulation indicated that the apparatus scaling-up without increasing the width of the gap between cylinders results in higher mechanical energy consumption per unit of processed starch slurry.
\end{abstract}

Keywords: starch gelatinization, starch granule swelling, rheological properties, Couette-Taylor flow

\section{INTRODUCTION}

Starch gelatinization, that occurs when starch slurry is heated, is a complex phase transition, during which starch partly dissolves in water (mostly amylose) and its granules (composed mostly of amylopectine) swell. One of the problems encountered during starch processing is a significant and rapid growth in the apparent viscosity of starch slurry. Additionally, in order to ensure good heat transfer conditions and to avoid separation of the starch from the water phase, an agitation of the slurry is needed (Sakonidou et al., 2003). In such a context, Couette-Taylor flow (CTF) apparatus was proposed for wheat starch processing (Hubacz and Buczyńska, 2011; Hubacz et al., 2013; Masuda et al., 2013). This type of apparatus consists of two cylinders with the inner one (rotor) rotating, while the fluid flows in the gap between these cylinders. When the angular velocity of the rotor exceeds a certain critical value the characteristic secondary fluid flow (Taylor vortices) arises, which intensifies fluid mixing and heat transfer conditions. Moreover, in the case of some non-Newtonian (shear-tinning) fluids the reduction in their apparent viscosity can be expected when rotor rotation increases. Additionally, it is worth pointing out that a CTF apparatus provides good environment for the 
intensification of various processes, e.g., multiple emulsion preparation (Dłuska and MarkowskaRadomska, 2010), processes involving gas-liquid flows (Dłuska and Hubacz, 2000), mass transfer in bioreactors (Ramezani et al., 2015), crystallization (Mayra and Kim, 2015).

During our previous studies on wheat starch gelatinization in a CTF apparatus (Hubacz and Buczyńska, 2011, Hubacz et al., 2013) the degree of starch gelatinization (DSG), starch granule swelling degree ( $S G S D$ ) and apparent viscosity of gelatinized starch were measured experimentally. The $D S G$, which is one of gelatinization indicators, was evaluated using the method of amylose-iodine complex formation proposed by Baks et al. (2007). Meanwhile, in order to measure the value of $S G S D$, a sample of gelatinized starch dispersion was centrifuged and the supernatant was separated from the swollen starch granules. The $S G S D$ was defined as the weight of the granules before freeze-drying to their weight after freeze-drying (Hubacz et al., 2013). It can be seen that SGSD quantifies the amount of water absorbed by starch granules, and because of that, $S G S D$ can be also related to the size increase of these granules during starch slurry thermal treatments.

The linear relationship between $S D S G$ and $D S G$ was found during starch processing in CTF apparatuses (Hubacz et al., 2013). However, the experiments (Hubacz et al., 2013) showed as well that, even if the gelatinization was completed $(D S G=1)$, starch granule swelling degree continued to change if the heating in a CTF apparatus was prolonged. This was connected to the starch pasting, which follows the gelatinization process (see e.g. Singh et al., 2009). Therefore, it can be said that the monitoring of starch granule swelling allows the monitoring of the progress of starch slurry thermal processing in a CTF apparatus for a wider range of the values of operating parameters than the monitoring of $D S G$. Nevertheless, the increase in the value of $S G S D$, which is not directly connected with the increase in starch granule diameter, may not be a perfect indicator of starch swelling. Moreover, the measurement of SGSD can be affected e.g. by the interstitial water in between starch granules, which could be difficult to remove from the sample using a centrifuge (Lagarrigue et al., 2008). Therefore, in order to estimate starch granule swelling during the heating, a starch granule size should be measured directly. Such an approach can be also supported by the fact that starch granule size distribution is one of the important parameters which affect the properties of starch dispersion after its thermal treatment. For example, the rheological properties of the gelatinized starch dispersion depend on that size distribution (Rao et al., 1997). However, gelatinized starch dispersion is a twophase system, therefore, its properties depend also on the concentration of dissolved starch in the liquid continuous phase in which the starch granules are suspended. It can be said that this dissolved starch is mostly in the form of amylose, because this polymer dissolves readily in water during the heating. Nevertheless, some shorter or less branched molecules of amylopectine can also be possibly found dissolved in the liquid phase after thermal treatment of starch slurry.

In this work, we studied changes in starch granule diameter distribution after the thermal treatment of starch slurry in a CTF apparatus. This was supplemented by the measurement of the concentration of starch dissolved in water during the process. This concentration was express by light absorbance $(A B S)$ in the liquid phase separated from the gelatinized starch dispersion. Additionally, such properties of starch dispersion like SGSD and its apparent viscosity were estimated.

The first objective of this work was the study how size distribution of starch granules and the values of $A B S$ or $S G S D$ are affected by experimental conditions in the CTF apparatus. The second objective was to find the relation between different parameters (i.e. average starch granule diameter, $A B S, S G S D$ and apparent viscosity) characterizing the properties of gelatinized starch dispersion. The third objective was to compare (using computer simulation of the process) the performance of the CTF apparatus used for this work with the performance of CTF apparatuses studied in the previous work (Hubacz et al., 2013). 


\section{MATERIALS AND METHODS}

\subsection{Starch origin and its properties}

During the experiments starch from wheat provided by Wako Pure Chemical Industries, Ltd. (193-13215) was used (12\% of moisture contents and $0.2-0.3 \%$ of protein). An average concentration of amylose and amylopectine in wheat starch was $25 \%$ and $75 \%$, respectively. The starch slurry was prepared in such a way that $1 \mathrm{dm}^{3}$ of the slurry contained $50 \mathrm{~g}$ of wheat starch. Therefore, when the moisture content was taken into account mass percentage of starch and water within the slurry was $4.4 \%$ and $95.6 \%$, respectively.

The motivations for this work were the problems encountered during cereal malt enzymatic hydrolysis, like high apparent viscosity of the fluid. Wheat starch represents just a simplification of such a system.

\subsection{Experimental conditions}

Starch was gelatinized in a Couette-Taylor flow (CTF) apparatus used by Masuda et al. (2013) for starch enzymatic hydrolysis (see Fig. 1). The apparatus was equipped with an outer water heat jacket, which was divided into two chambers in order to improve the control of temperature (important in the case of starch hydrolysis). However, during the present study water of the same temperature was pumped into both chambers of the heat jacket. The length of the CTF apparatus used by us was $0.3 \mathrm{~m}$, while the radii of the inner and outer cylinder were 0.0125 and $0.0175 \mathrm{~m}$, respectively.

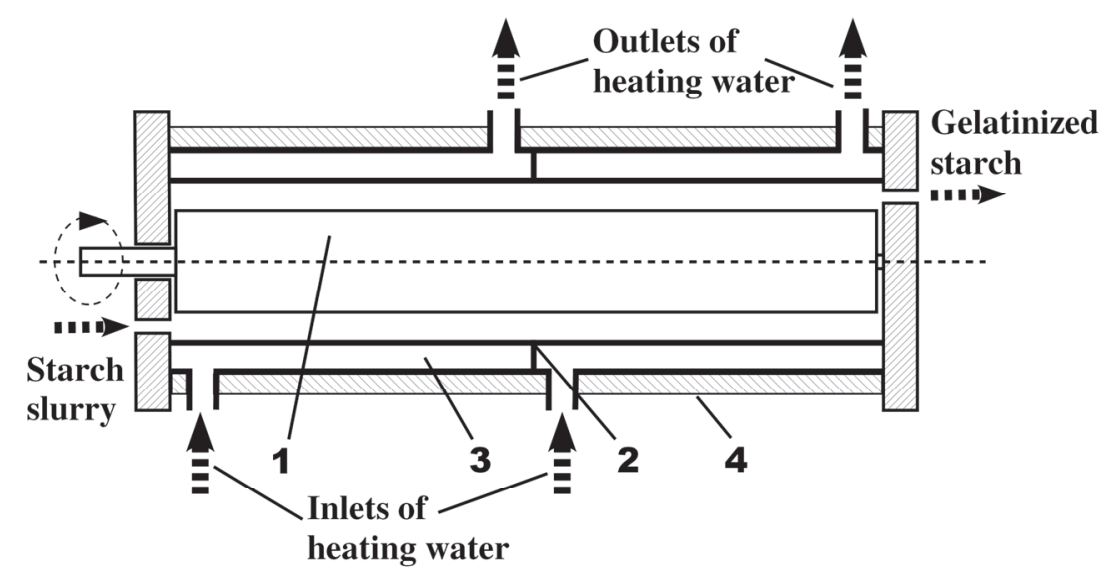

Fig. 1. Sketch of Couette-Taylor flow apparatus; 1-rotor, 2-outer cylinder, 3-heat jacket, 4- isolation

The conditions of thermal treatment in the CTF apparatus were specified by the rotor angular velocity $\omega(10.04,20.09$ or $30.13 \mathrm{rad} / \mathrm{s})$, the temperature of water in the heat jacket $T_{h j}\left(65,70,85\right.$ or $\left.90{ }^{\circ} \mathrm{C}\right)$ and the starch slurry axial velocity $u(0.000531$ or $0.000743 \mathrm{~m} / \mathrm{s})$. The starch slurry axial velocity was defined as:

$$
u=Q / \pi\left(R_{2}^{2}-R_{1}^{2}\right)
$$

where $Q$ is the volumetric flow rate of the starch slurry measured at the inlet conditions. Each experiment, specified by the same experimental conditions, was repeated 3 times.

The applied values of the temperature of water in the heat jacket roughly correspond to the gelatinization (about $56-65^{\circ} \mathrm{C}$ ) and pasting temperatures (about $82^{\circ} \mathrm{C}$ ) of wheat starch (see Sigh et al, 
2009). It is also worth underlining that during our study we were interested in the investigation how operating parameters influence starch thermal treatment in a CTF apparatus. Meanwhile, the production of gelatinized starch, which may require even higher temperature from the technological point of view, was less important for us. Moreover, because Patel and Seetharaman (2006) reported problems during the analysis of the pictures of wheat starch granules after their treatment at the temperature above $70{ }^{\circ} \mathrm{C}$, the measurements of starch granule diameter were limited to the experiments carried out at $T_{h j} \leq 70{ }^{\circ} \mathrm{C}$. A similar approach was applied by other researchers (Malumba et al., 2013 or Patel and Seetharaman, 2006).

\subsection{Sample handling}

In order to obtain an averaged representation of the starch dispersion after treatment in the CTF apparatus a sample of at least a few $\mathrm{ml}$ was taken at the outlet of the apparatus. For this purpose an aluminium vessel (with a thin wall) was used. The outside of the vessel was covered with a layer of ice (1 cm thick), whose temperature was about $-5{ }^{\circ} \mathrm{C}$ (Hubacz and Buczyńska 2011). The sample taking procedure was a continuous process, during which starch dispersion gathered in the vessel was gently stirred and mixed with a new portion of the dispersion of high temperature. This allowed reduction of the temperature of the sample below the temperature of gelatinization, while keeping this temperature well above $0{ }^{\circ} \mathrm{C}$ (around $20-25^{\circ} \mathrm{C}$ ). Subsequently, the sample was analysed within a few minutes.

\subsection{Starch granule size measurement}

The sample was examined using a digital microscope (VHX-100, Keyence Co., Ltd.), with which the software for image analysis was provided (Particle Analysis Ver. 3.5, Nippon Steel and Sumitomo Metal Technology Co., Ltd.). The microscope observation and picture registration was carried out at ambient temperature (about $25^{\circ} \mathrm{C}$ ). During the picture analysis, the area occupied by each granule was measured and the diameter of each granule was calculated assuming that the images of the granules can be approximated by circles. The measurement of the area occupied by granule images and the counting of granule numbers were done using a manual mode. This tedious procedure was chosen because some problems with a correct recognition of the granule borders were noticed during automatic working mode of the software. For each experimental condition, the size of 8500 (about 25 pictures) starch granules was measured on average. As a result the granule diameter distribution, mean diameter and standard deviation of granule population were calculated for the given experimental condition.

\subsection{Starch solubility and SGSD measurements}

To evaluate how much starch dissolved in water during the starch slurry thermal treatment in the CTF apparatus a sample of gelatinized starch was centrifuged at $2000 \mathrm{~g}$ for $30 \mathrm{~min}$ (to separate solid from liquid phase). The supernatant was 20 times diluted and $1.33 \mathrm{ml}$ of it was mixed with $5 \mathrm{ml}$ of aqueous solution of $0.20 \%$ I and $0.66 \% \mathrm{KI}$. Subsequently, distilled water was added up to the volume of $20 \mathrm{ml}$. Finally, the absorbance of the light (wavelength $630 \mathrm{~nm}$ ) in the sample was measured with a UV-vis spectrophotometer (Shimadzu Co. Ltd.). It was assumed that the value of the light absorbance (ABS) was proportional to the dissolved starch concentration in the supernatant. Although it was believed that dissolved starch was mostly in the form of amylose, some shorter or less branched molecules of amylopectine can be also possibly detected using this method. It is worth remembering that this procedure should not be confused with procedure of DSG measurement (Baks et al. 2007; Hubacz and Buczyńska, 2011), which was also based on the amylose-iodine complex formation. The concentration of amylose (or dissolved starch) in the supernatant was the result of amylose leaching from the starch granules and the water absorption by the granules (i.e. granule swelling). Meanwhile, the DSG 
procedure was related only to amylose leaching during gelatinization but did not take into account how much water was absorbed by starch granules during gelatinization.

The value of SGSD was evaluated using the sediment separated from starch dispersion during centrifugation. The wet sediment was weighed, dried in a freeze-dryer (using Eyela Freeze Dryer FD-1000) and once again weighed. The value of SGSD was defined as the weight of the sediment before freeze-drying to its weight after freeze-drying.

\subsection{Measurements of starch paste apparent viscosity}

The rheological properties of gelatinized starch were measured using a concentric cylinder viscometer (Haake Viscotester 550, Thermo Fisher Scientific Inc.). The sketch of the viscometer was shown in Fig.2. The width of the gaps between cylinders was 350 and $400 \mu \mathrm{m}$, which was a few times bigger than the diameter of starch granules in the dispersion (at least in the cases for which the measurement of starch granule diameter was carried out). The measurement of viscosity was conducted at $T=25{ }^{\circ} \mathrm{C}$, which was approximately the temperature of starch sample after its cooling using the aforementioned procedure. The volume of the sample needed for the measurement was $9 \mathrm{ml}$. During the measurement the shear rate was changed from 110 to 1999 1/s and subsequently, from 1999 to 100 1/s, while the total duration of the measurement was $406 \mathrm{~s}$.

According to the manual the viscosity range which can be detected using this viscometer was 2 - 2000 Pas. The apparent viscosity of starch dispersion examined in the present study was inside this range. Moreover, the calculation of the rotational Reynolds number for the lowest measured value of apparent viscosity at the highest value of applied sheer rate confirmed that the flow in viscometer was laminar without Taylor vortices. However, in the case of gelatinized starch dispersion obtained during the experiments carried out at $T_{h j} \leq 70{ }^{\circ} \mathrm{C}$ the points of the flow curve were rather scattered at low values of shear rate (below approximately 1000 1/s), which suggested high experimental uncertainties at that range of shear rate. Therefore, in the case of such starch dispersion the parameters of the powerlaw model were estimated only based on the measurement obtained at the values of shear rate higher than about $1000 \mathrm{1} / \mathrm{s}$. On the contrary, in the case of gelatinized starch dispersion obtained during the experiments carried out at $T_{h j} \geq 85^{\circ} \mathrm{C}$ the apparent viscosity was high enough, so that a smooth flow curve could be obtained for the whole range of the shear rate values. Therefore, in the case of such starch dispersion no point forming this flow curve was rejected during the estimation of the parameters of the power-law model.

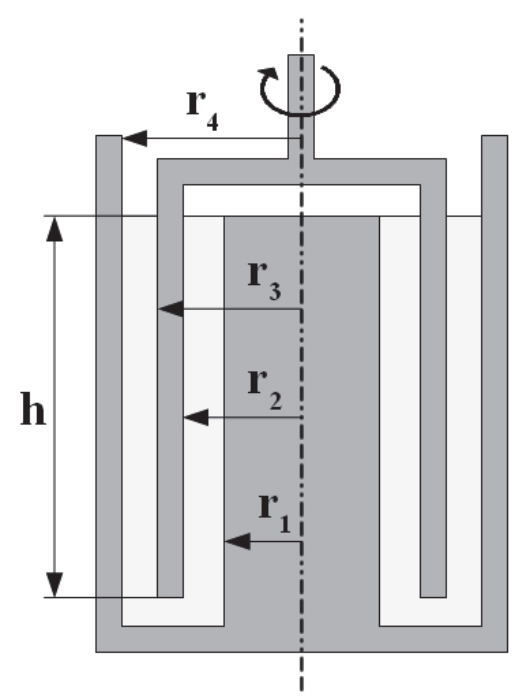

Fig. 2. Sketch of the viscometer geometry;

$r_{4}=20.5 \mathrm{~mm} ; r_{3}=20.1 \mathrm{~mm} ; r_{2}=17.85 \mathrm{~mm} ; r_{1}=17.5 \mathrm{~mm} ; h=60 \mathrm{~mm}$ 


\subsection{CFD Simulation}

CFD simulation was used in order to compare gelatinization in the studied CTF apparatus with the other two apparatuses studied by Hubacz et al. (2013). The size of the latter apparatuses was defined by the radius of outer cylinder $R_{2}=0.026 \mathrm{~m}$, the radius of rotor $R_{1}=0.016 \mathrm{~m}$ or $0.021 \mathrm{~m}$, and the length $L=0.3 \mathrm{~m}$. This comparison can provide interesting information how to scale-up or intensify the process by changing the geometry of a CTF apparatus. On the other hand, such a comparison through only experimental results could be difficult because numerical simulations provide additional information difficult to obtain through experiments like hydrodynamics of the flow in a CTF apparatus or mechanical power demand for the process. This information is, however, important for the comparison of the apparatus performance.

The hydrodynamics of the flow during starch gelatinization was calculated using steady state NavierStokes equations adapted for a 2D axisymmetric swirl model (see Hubacz and Buczyńska, 2011). The 2D axisymmetric swirl model is based on the assumption that there is no circumferential gradient in the flow, although, there may be non-zero circumferential or swirl velocity. In addition to this, energy and species transport equations were solved (see Hubacz and Buczyńska, 2011). These equations were included into the model in order to calculate rheological properties of starch slurry, which was modelled using a power-law equation (see Hubacz et al., 2013):

$$
\mu=(0.016 D S G+0.0383) j^{-0.35}
$$

where, $\mu$ is the fluid apparent viscosity and $\dot{\gamma}$ is the shear rate. Nevertheless, it should be remember that Eq. 2 represents rather a simplified approach to the problem, which enables only rough prediction of apparent viscosity of the fluid during the investigated problem.

During the simulation the degree of starch gelatinization was defined using the following equation:

$$
D S G=\frac{X_{s g}}{X_{s 0}}
$$

where $X_{s g}$ is the mass fraction of gelatinized starch, and $X_{s 0}$ is the total mass fraction of starch in the slurry. The other fluid properties (density, heat capacity, thermal conductivity, diffusion (dispersion) coefficient were assumed to be constant $\left(\rho=998.2 \mathrm{~kg} / \mathrm{m}^{3}, C_{p}=4.182 \mathrm{~J} /(\mathrm{kg} \cdot \mathrm{K}), \lambda=0.6 \mathrm{~W} /(\mathrm{m} \cdot \mathrm{K})\right.$, $D_{j}=10^{-13} \mathrm{~m}^{2} / \mathrm{s}$ ). Finally, the kinetics of starch gelatinization was modelled using the first order equation proposed in Brandam et al. (2003).

During the simulation it was assumed, that the temperature of the outer cylinder was between $60{ }^{\circ} \mathrm{C}$ and $85^{\circ} \mathrm{C}$, while the inlet temperature of starch slurry was $20^{\circ} \mathrm{C}$. The axial velocity of starch slurry inside the apparatus was assumed to be between 0.00033 and $0.00099 \mathrm{~m} / \mathrm{s}$. Finally, it was assumed that the surface of the rotor was an adiabatic wall (no heat transfer through it) which rotated with the angular velocity $\omega$ (between 5.78 and $19.27 \mathrm{rad} / \mathrm{s}$ ). However, because of a possibility of "back flows" at the outlet from the apparatus, the calculations were carried out for the length of the apparatus which equalled $0.6 \mathrm{~m}$. At the location between 0.4 and $0.6 \mathrm{~m}$ from the inlet to the apparatus no rotor rotation was imposed. The calculations were conducted assuming that the axial gradients of variables at the outlet plane are zero. No significant influence of the outlet boundary conditions on the results was observed at the distance of $0.3 \mathrm{~m}$ from the inlet of the apparatus (it corresponds to the length of the experimental apparatus).

Calculations were carried out using Ansys 14.5 (Fluent) computer code assuming laminar flow due to high apparent viscosity of gelatinized starch. The second order upwind scheme was used to discretize the equations. The pressure-velocity coupling was performed using the SIMPLE scheme. Grids with a cell number 24000 and 96000 were used during the simulations, with no significant deference in the obtained results. 
Because the applied rheological model of the fluid is a rather rough approximation of starch slurry properties during its thermal treatments, the model of starch gelatinization described in this chapter represents a rather simplistic approximation of the process. Nevertheless, it enabled judgment how the non-Newtonian properties of the fluid could influence starch gelatinization (Hubacz et al., 2013). Moreover, this model was used for the numerical simulations of starch gelatinization in two concentric CTF apparatuses of different gap width between cylinders (Hubacz et al., 2013). Good agreement between these simulations and the experimental results was achieved. Therefore, it was assumed that this model is good enough for the comparison of CTF apparatuses of different geometries and the preliminary flow hydrodynamics prediction inside of such an apparatus during starch gelatinization.

\section{RESULTS}

\subsection{Starch granule size distribution after treatment in CTF apparatus}

The granules of native starch are shown in Fig. 3a, while their size distribution can be seen in Fig. 4. This size distribution was used to calculate the average granule diameter $\left(D_{0}=20.63 \mu \mathrm{m}\right)$ and standard deviation $\left(S D_{0}=8.11 \mu \mathrm{m}\right)$ for the native starch granule population.
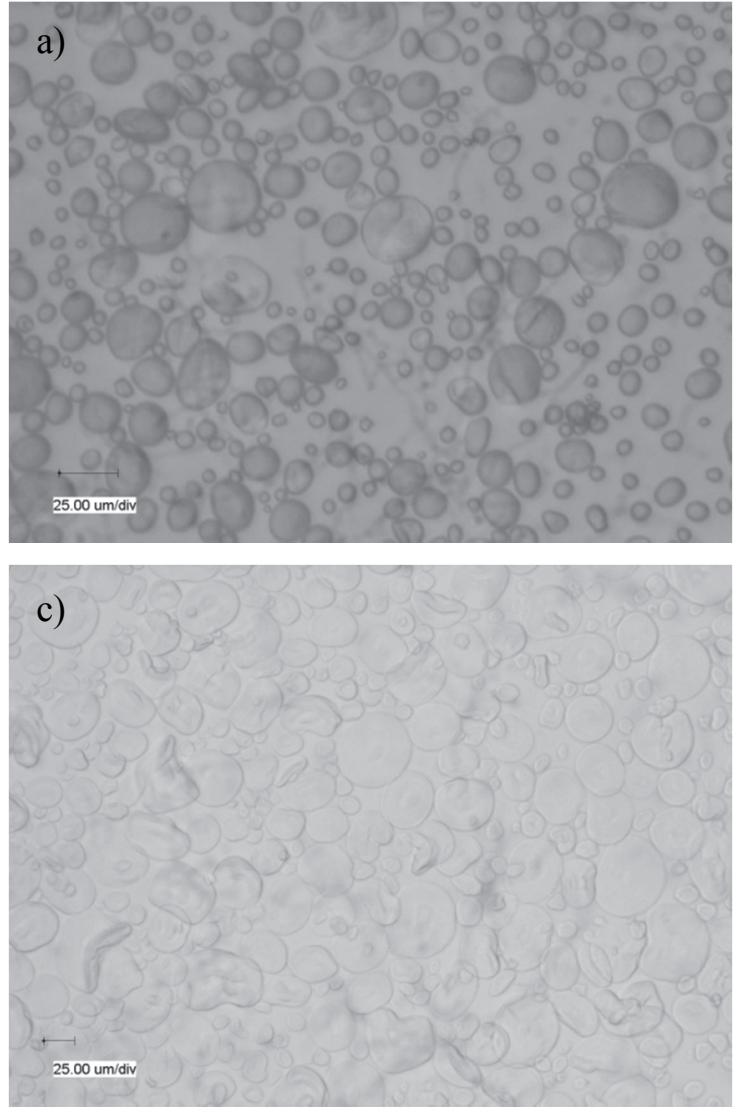
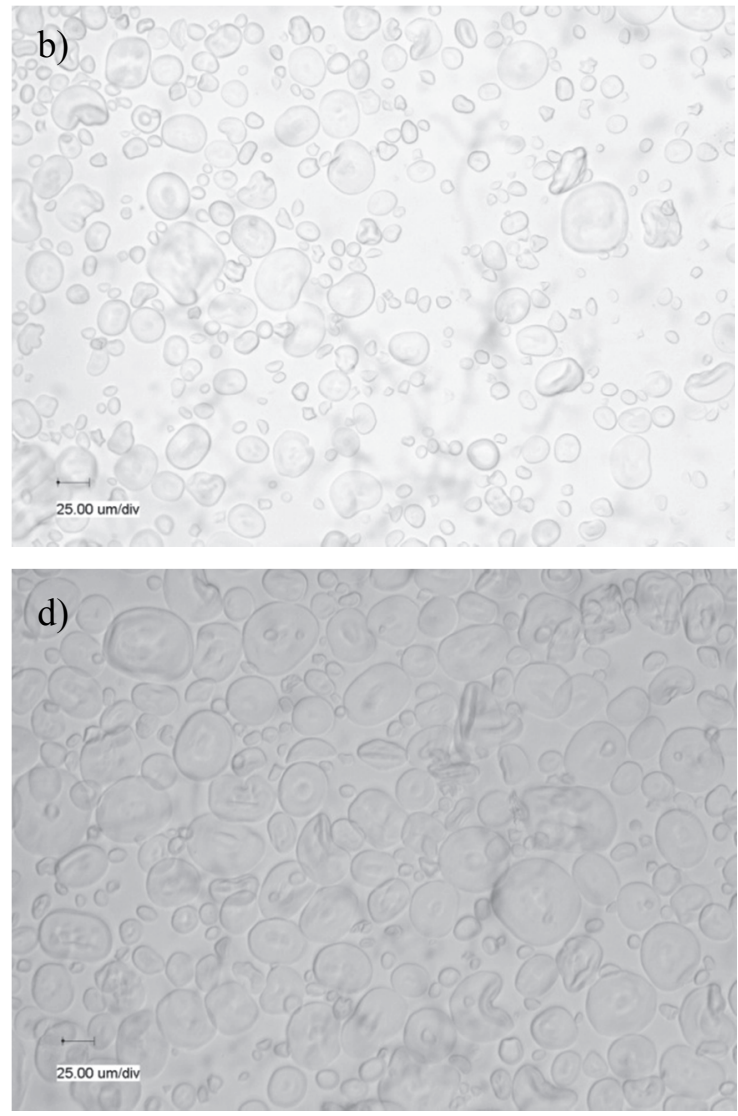

Fig. 3. Native starch (the picture is about 2 times bigger scale than the other pictures); starch granules after treatment in the following conditions: b) $\left.u=0.000743 \mathrm{~m} / \mathrm{s}, \omega=10.04 \mathrm{rad} / \mathrm{s}, T_{h j}=65^{\circ} \mathrm{C}, \mathrm{c}\right) u=0.000743 \mathrm{~m} / \mathrm{s}$, $\left.\omega=10.04 \mathrm{rad} / \mathrm{s}, T_{h j}=70^{\circ} \mathrm{C}, \mathrm{d}\right) u=0.000531 \mathrm{~m} / \mathrm{s}, \omega=20.09 \mathrm{rad} / \mathrm{s}, T_{h j}=70^{\circ} \mathrm{C}$ (from Hubacz et al. 2016)

The examples of starch granule appearance after their treatment in the CTF apparatus at different conditions are shown in Figs. 3b-d (the scale of these pictures is about 2 times smaller than the scale of the picture in Fig. 3a), while the examples of granule size distributions of gelatinized starch are 
presented in Fig.4. In addition to this, the values of the average granule diameters of gelatinized starch $(D)$ and corresponding standard deviations $(S D)$ are given in Table 1. Both the pictures in Fig. 3 and the data from Table 1 indicate a significant increase in starch granule diameters after its thermal treatment. Similarly, the value of standard deviation of granule population increased after the processing in the CTF apparatus. This agreed with other authors' observation, that gelatinization causes an increase in granule diameter and standard deviation (see e.g. Rao et al., 1997).

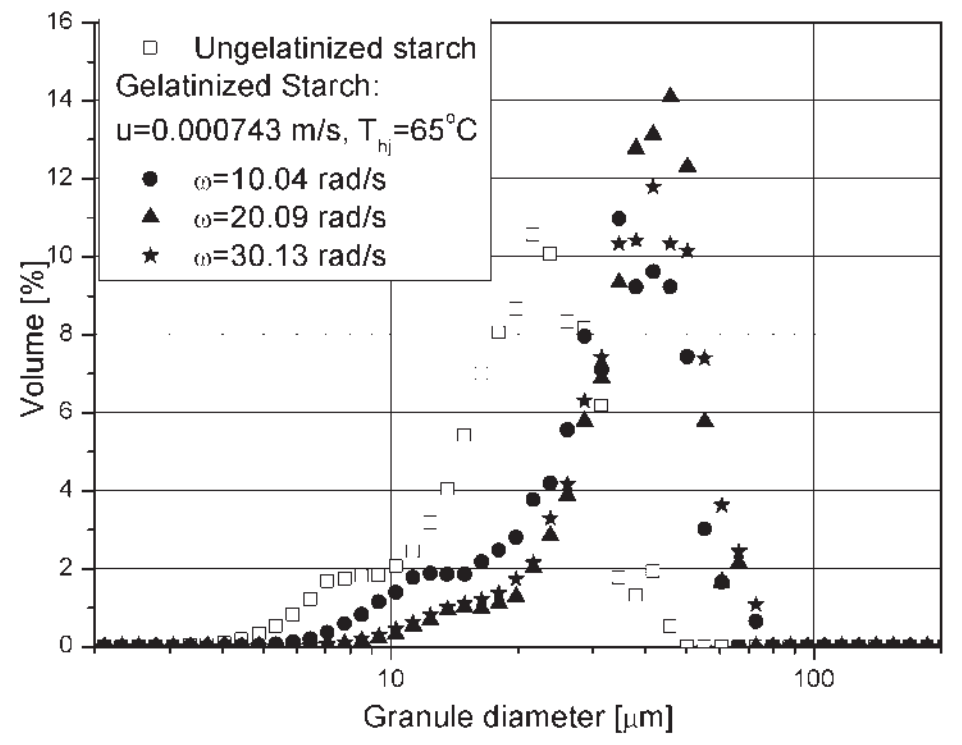

Fig. 4. Distribution of starch granule diameter after treatment in CTF apparatus (from Hubacz et al. 20016)

Table 1. Average starch granule diameter $(D)$ and standard deviation of granule size distribution $(S D)$ after treatment in CTF apparatus (for ungelatinized starch $D_{0}=20.63 \mu \mathrm{m}$ and $S D_{0}=8.11 \mu \mathrm{m}$ ) (from Hubacz et al., 2016)

\begin{tabular}{|c|c|c|c|c|}
\hline $\begin{array}{c}\omega \\
\mathrm{rad} / \mathrm{s}\end{array}$ & $\begin{array}{c}u \\
\mathrm{~m} / \mathrm{s}\end{array}$ & $\begin{array}{c}T_{h \mathrm{j}} \\
{ }^{\circ} \mathrm{C}\end{array}$ & $\begin{array}{c}D \\
\mu \mathrm{m}\end{array}$ & $\begin{array}{c}S D \\
\mu \mathrm{m}\end{array}$ \\
\hline 10.04 & 0.000743 & 65.0 & 32.94 & 13.08 \\
\hline 20.09 & 0.000743 & 65.0 & 38.86 & 11.64 \\
\hline 30.13 & 0.000743 & 65.0 & 38.82 & 13.11 \\
\hline 10.04 & 0.000743 & 70.0 & 42.18 & 15.32 \\
\hline 20.09 & 0.000743 & 70.0 & 43.57 & 14.93 \\
\hline 30.13 & 0.000743 & 70.0 & 45.36 & 14.97 \\
\hline 10.04 & 0.000531 & 70.0 & 42.26 & 14.77 \\
\hline 20.09 & 0.000531 & 70.0 & 45.61 & 14.44 \\
\hline
\end{tabular}

The impact of the operating parameters on $D$ and $S D$ during the treatment in the CTF apparatus is illustrated in Table 1 . The values of $D$ and $S D$ were significantly affected only by $T_{h j}$ : higher gelatinization temperature results in higher values of both parameters. On the other hand, the influence of $u$ and $\omega$ on $D$ or $S D$ was significantly less pronounced. Therefore, in order to verify whether or not the impact of $u$ and $\omega$ on starch granule size distribution can be neglected the Kolmodorov-Smirnov test was done. This test relies on finding the biggest difference between cumulative distribution functions of two compared populations. In this case there are just two populations of starch granule diameters $\left(D_{i}\right)$ obtained at different experimental conditions. Those populations are characterized by cumulative distribution functions (respectively $F_{n 1}\left(D_{i}\right)$ and $F_{n 2}\left(D_{i}\right)$ ) and by the number of granules in each population (respectively $n_{1}$ and $n_{2}$ ). The results of the test are shown in Table 2 . 
In the last column of Table 2 there are shown values of a parameter which is a product of two other parameters defined by the equations:

$$
\begin{gathered}
n=\sqrt{\frac{n_{1} n_{2}}{n_{1}+n_{2}}} \\
\delta_{n 1, n 2}=\sup _{D_{i}}\left|F_{n 1}\left(D_{i}\right)-F_{n 2}\left(D_{i}\right)\right|
\end{gathered}
$$

where, $\delta_{n 1, n 2}$ is the biggest difference between the cumulative distribution functions of two compared populations of starch granule diameters. In the first five rows of Table 2 there are shown comparisons of granule diameter distributions obtained at different values of $\omega$ but with the same values of other operating parameters. Meanwhile, rows 6 and 7 are the comparisons of granule diameter distributions obtained at different values of $u$ but with the same values of other operating parameters. When assuming the value of statistical significance at the level of 0.05 , two population of granules are characterized by the same diameter distribution if $n \cdot \delta_{n 1, n 2}<1.36$ (see e.g. Hellwig, 1998). Nevertheless, in all the cases presented in Table 2 this condition is not fulfilled. Therefore, it can be stated that the impact of the values of $\omega$ and $u$ on the distribution of starch granule diameter after a treatment in the CTF apparatus cannot be neglected: the average diameter of gelatinized starch granules increased when the axial velocity decreased and when the rotor angular velocity increased.

Table 2. The results of Kolmogorov-Smirnov Test

\begin{tabular}{|c|c|c|c|c|c|c|c|}
\hline \multirow{2}{*}{ No. } & \multicolumn{3}{|c|}{ Granule population 1} & \multicolumn{3}{c|}{ Granule population 2} & \multirow{2}{*}{$n \cdot \delta_{n 1, n 2}$} \\
\cline { 2 - 7 } & $\begin{array}{c}\omega \\
\mathrm{rad} / \mathrm{s}\end{array}$ & $\begin{array}{c}u \\
\mathrm{~m} / \mathrm{s}\end{array}$ & $\begin{array}{c}T_{h \mathrm{j}} \\
{ }^{0} \mathrm{C}\end{array}$ & $\begin{array}{c}\omega \\
\mathrm{rad} / \mathrm{s}\end{array}$ & $\begin{array}{c}u \\
\mathrm{~m} / \mathrm{s}\end{array}$ & $\begin{array}{c}T_{h \mathrm{j}} \\
{ }^{0} \mathrm{C}\end{array}$ & \\
\hline 1 & 10.04 & 0.000743 & 65.0 & 20.09 & 0.000743 & 65.0 & 16.31 \\
\hline 2 & 30.13 & 0.000743 & 65.0 & 20.09 & 0.000743 & 65.0 & 3.06 \\
\hline 3 & 10.04 & 0.000743 & 70.0 & 20.09 & 0.000743 & 70.0 & 2.23 \\
\hline 4 & 30.13 & 0.000743 & 70.0 & 20.09 & 0.000743 & 70.0 & 2.61 \\
\hline 5 & 10.04 & 0.000531 & 70.0 & 20.09 & 0.000531 & 70.0 & 7.92 \\
\hline 6 & 10.04 & 0.000743 & 70.0 & 10.04 & 0.000531 & 70.0 & 2.53 \\
\hline 7 & 20.09 & 0.000743 & 70.0 & 20.09 & 0.000531 & 70.0 & 4.8 \\
\hline
\end{tabular}

The significant increase in $D$ (it can be seen especially in the case of $T_{h j}=65^{\circ} \mathrm{C}$ ) when the value of $\omega$ was changed from 10.04 to $20.09 \mathrm{rad} / \mathrm{s}$ may suggest that the transition to the Taylor vortex flow in the CTF apparatus (in the investigated system) occurs at a value of $\omega$ lower than $20.09 \mathrm{rad} / \mathrm{s}$ but higher than $10.04 \mathrm{rad} / \mathrm{s}$. This hypothesis stems from the fact that the transition to the vortex flow pattern denotes the intensification of heat transfer in the apparatus. This statement was verified using computer simulations of starch gelatinization in the CTF apparatus, which revealed that the Taylor vortex flow pattern had been gradually developed with increasing value of $\omega$. At $\omega=10.04 \mathrm{rad} / \mathrm{s}$ the computations indicated laminar flow without vortices along the whole length of the apparatus. At $\omega=12.04 \mathrm{rad} / \mathrm{s}$, only a vortex pair occurred close to the apparatus inlet, but in the farther part of the apparatus it disappeared due to the increase in the fluid apparent viscosity. At $\omega=15 \mathrm{rad} / \mathrm{s}$ the vortex pattern could be seen along the significant part of the apparatus. However, only when $u=0.000743 \mathrm{~m} / \mathrm{s}$ and $T_{h j}=65^{\circ} \mathrm{C}$ the increasing viscosity did not suppress the Taylor vortices up to the distance of about $0.3 \mathrm{~m}$ from the inlet (corresponding to the length of the experimental apparatus). Finally, when the calculations were conducted at $\omega=17.5 \mathrm{rad} / \mathrm{s}$ the vortex flow pattern was developed along the whole length of the apparatus independently of the values of $u$ and $T_{h j}$. To summarize, the results of the simulations seem to support the hypothesis that the transition to the Taylor vortex flow could occur for the value of $\omega$ between 10.04 and $20.09 \mathrm{rad} / \mathrm{s}$. Nevertheless, the results of the computer simulations 
should be considered with caution, because the model of the process used for the simulations was rather simplified.

\subsection{Properties of gelatinized paste}

In Table 3 there are shown the values of starch granule swelling degree of gelatinized starch dispersion after treatment in the CTF apparatus. For the same experimental conditions the concentrations of starch dissolved in water were also estimated. The starch concentration was expressed by the value of light absorbance ( $A B S$ - see also Table 3 ) in a sample prepared using the water separated from the granules of the gelatinized starch (procedure described above): a higher value of $A B S$ denoted higher concentration of dissolved amylose.

Table 3. Starch granule swelling degree and light absorbance by the continuous phase of starch dispersion

\begin{tabular}{|c|c|c|c|c|}
\hline $\begin{array}{c}u \\
\mathrm{~m} / \mathrm{s}\end{array}$ & $\begin{array}{c}\omega \\
\mathrm{rad} / \mathrm{s}\end{array}$ & $\begin{array}{c}T_{h j} \\
{ }^{\circ} \mathrm{C}\end{array}$ & SGSD & ABS \\
\hline 0.000743 & 10.04 & 65 & 5.27 & 0.064 \\
\hline 0.000743 & 20.09 & 65 & 5.16 & 0.082 \\
\hline 0.000743 & 30.13 & 65 & 5.18 & 0.086 \\
\hline 0.000743 & 10.04 & 70 & 6.00 & 0.110 \\
\hline 0.000743 & 20.09 & 70 & 6.03 & 0.127 \\
\hline 0.000743 & 30.13 & 70 & 6.10 & 0.134 \\
\hline 0.000531 & 10.04 & 70 & 6.13 & 0.139 \\
\hline 0.000531 & 20.09 & 70 & 6.07 & 0.156 \\
\hline 0.000531 & 30.13 & 70 & 6.14 & 0.152 \\
\hline 0.000531 & 10.04 & 85 & 8.02 & 0.336 \\
\hline 0.000531 & 20.09 & 85 & 8.06 & 0.339 \\
\hline 0.000531 & 30.13 & 85 & 8.23 & 0.346 \\
\hline 0.000531 & 10.04 & 90 & 9.04 & 0.485 \\
\hline 0.000531 & 20.09 & 90 & 9.40 & 0.529 \\
\hline 0.000531 & 30.13 & 90 & 9.63 & 0.565 \\
\hline
\end{tabular}

The value of $A B S$ depended on the values of operating parameters in the same manner like in the case of $D$ (Tables 1 and 3). Temperature in the heat jacket affected light absorbance in the most significant way: the increase in temperature caused the growth in the value of $A B S$. The value of $A B S$ also grew when angular velocity of rotor increased and axial velocity decreased. This observation suggested that the correlation between $A B S$ and $D$ can be found. Moreover, similarly like in the case of $D$ a significant increase in the value of ABS was observed when the value of $\omega$ was changed from 10.04 to $20.09 \mathrm{rad} / \mathrm{s}$. This seems to confirm the suggestion that the transition to the Taylor vortex flow in the CTF apparatus (in the investigated system) occurs at a value of $\omega$ lower than $20.09 \mathrm{rad} / \mathrm{s}$ but higher than $10.04 \mathrm{rad} / \mathrm{s}$.

On the contrary, a similar impact of operating parameters on $S D S G$ like in the case of $A B S$ and $D$ could be seen only for experiments carried out at $T_{h j} \geq 85^{\circ} \mathrm{C}$ (see Table 3). When the heat jacket temperature was smaller the impact of this temperature on $S G S D$ was still significant, but the changes in $\omega$ or $u$ seemed to have little influence on $S G S D$ (Table 3). One of the possible explanations of those observations could be the fact that different phenomena dominate the process at $T_{h j} \leq 70^{\circ} \mathrm{C}$ and at $T_{h j} \geq 85^{\circ} \mathrm{C}$. When the experiments were carried out at $T_{h j} \leq 70^{\circ} \mathrm{C}$ the early stage of starch slurry processing probably preponderated during the treatment in the CTF apparatus. Such an early stage of starch processing denoted that gelatinization of starch was not completed and water absorption by 
starch granules was accompanied by e.g. amylose leaching. Therefore, the weight gain of starch granules caused by water absorption, which can be measured by $S G S D$, could be reduced to some extend by amylose leaching from the granules. Meanwhile, both amylose leaching and water absorption favored the increase in dissolved starch concentration (represented by $A B S$ ) in the continuous water phase in which starch granules were suspended. On the contrary, at $T_{h j} \geq 85^{\circ} \mathrm{C}$ gelatinization can be completed $(D S G=1)$ even for higher values of starch slurry axial flow and values of $\omega$ lower than those investigated here (see Hubacz et al., 2013 and Masuda et al., 2013). When gelatinization is completed a pasting process starts and water absorption by starch granules (i.e. granule swelling) dominates that process. Under such conditions starch granule swelling, water absorption and the increase in the concentration of dissolved starch should be related with each other.

Nevertheless, in the case of experiments conducted at $T_{h j} \leq 70{ }^{\circ} \mathrm{C} D$ increased when $\omega$ increased, while the value of $S G S D$ did not seem to depend very significantly on the rotor angular velocity. This suggested that starch granule swelling (represented by the changes in $D$ ) was independent from water absorption by these granules (represented by $S G S D$ ) at that range of process temperature. This in turn is difficult to understand. Therefore, the weak impact of $\mathrm{u}$ and $\omega$ on $S G S D$ at $T_{h j} \leq 70^{\circ} \mathrm{C}$ could just result from the $S G S D$ measurement procedure. For example the impact of the interstitial water in between starch granules (which could be difficult to remove from the sample using a centrifuge) on the value of $S G S D$ could be more significant when $T_{h j} \leq 70{ }^{\circ} \mathrm{C}$ than when $T_{h j} \geq 85^{\circ} \mathrm{C}$. This stemmed from the fact that the amount of water absorbed by the granules was lower at the lower value of temperature.

Furthermore, the impact of $\omega$ on SGSD seemed to be different than that observed in the case of our previous studies (Hubacz et al., 2013). The results from Hubacz et al. (2013) revealed an increase in $S G S D$ with increasing $\omega$ for $\omega<11.56 \mathrm{rad} / \mathrm{s}$ and a reversed tendency for $\omega>11.56 \mathrm{rad} / \mathrm{s}$. Meanwhile, in the present experiments the impact of $\omega$ on $S G S D$ was difficult to evaluate for $T_{h j} \leq 70^{\circ} \mathrm{C}$, while for $T_{h j} \geq 85^{\circ} \mathrm{C}$ the increase in $S G S D$ with the increasing $\omega$ (without changing the tendency) was observed. The reason of this discrepancy cannot be easily found using the available experimental data, because the CTF apparatuses used by Hubacz et al. (2013) were bigger than that the one used in our experiment. This means that the transitions between flow regimes occur at the higher $\omega$ in the case of the apparatus used for the present study than in the case of the apparatuses used previously (Hubacz et al., 2013). Therefore, a similar impact of $\omega$ on granule swelling could be observed if the present experiments were carried out for the wider range of $\omega$ than it was done so far.

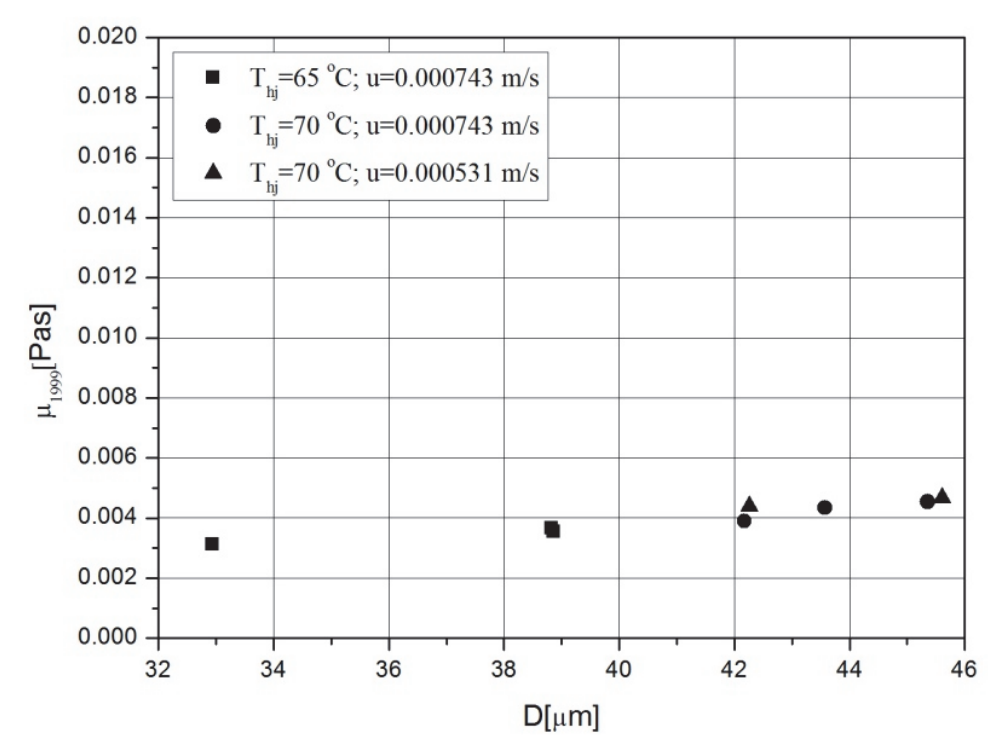

Fig. 5. Dependence of $\mu_{1999}$ on average starch granule diameter, $\mu_{1999}$ apparent viscosity of gelatinized starch paste at the maximum shear rate (1999 1/s) 
The dependences of starch dispersion apparent viscosity measured at the highest value of shear rate (1999 1/s) on D, SDSG and $A B S$ are shown in Figs. 5 - 7. The correlations between these parameters and $\mu_{1999}$ are relatively good. This agreed with the results presented in Hubacz et al. (2013), in which good correlation of gelatinized starch apparent viscosity with SGSD was observed. Moreover, this seems to confirm that the values of $D, S D S G$ or $A B S$ convey similar information on the state of gelatinized starch paste. Nevertheless, when only experimental results obtained at $T_{h j} \leq 70^{\circ} \mathrm{C}$ were analysed the correlation between $\mu_{1999}$ and $S G S D$ was a little worse. This (as aforementioned) is probably connected with the fact that $S G S D$ did not depend significantly on the rotor angular velocity when the experiments were conducted at $T_{h j} \leq 70^{\circ} \mathrm{C}$.

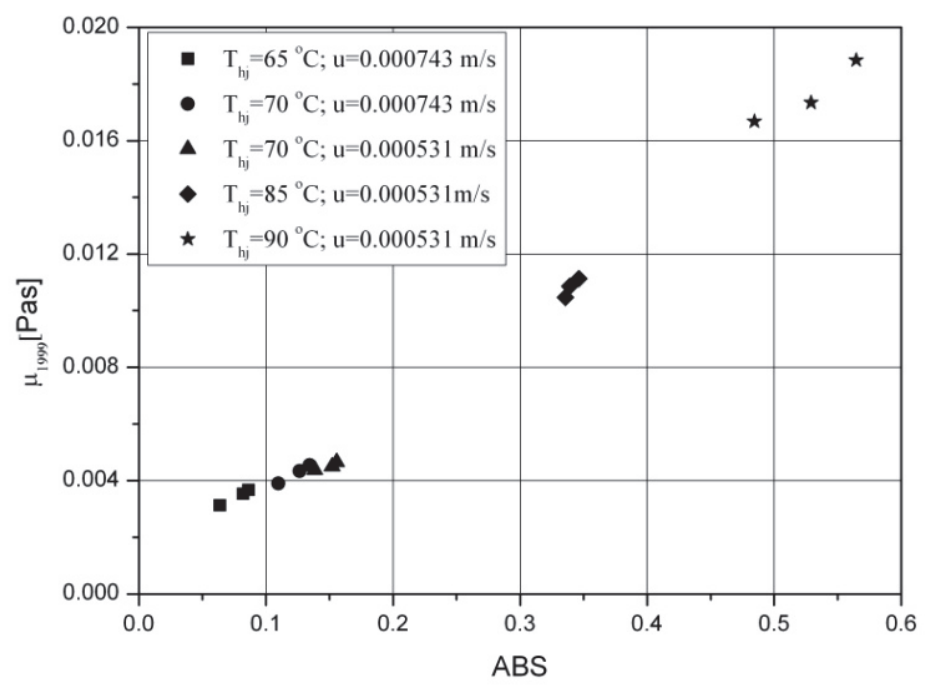

Fig. 6. Dependence of $\mu_{1999}$ on $A B S-\mu_{1999}$ apparent viscosity of gelatinized starch paste at the maximum shear rate (1999 1/s)

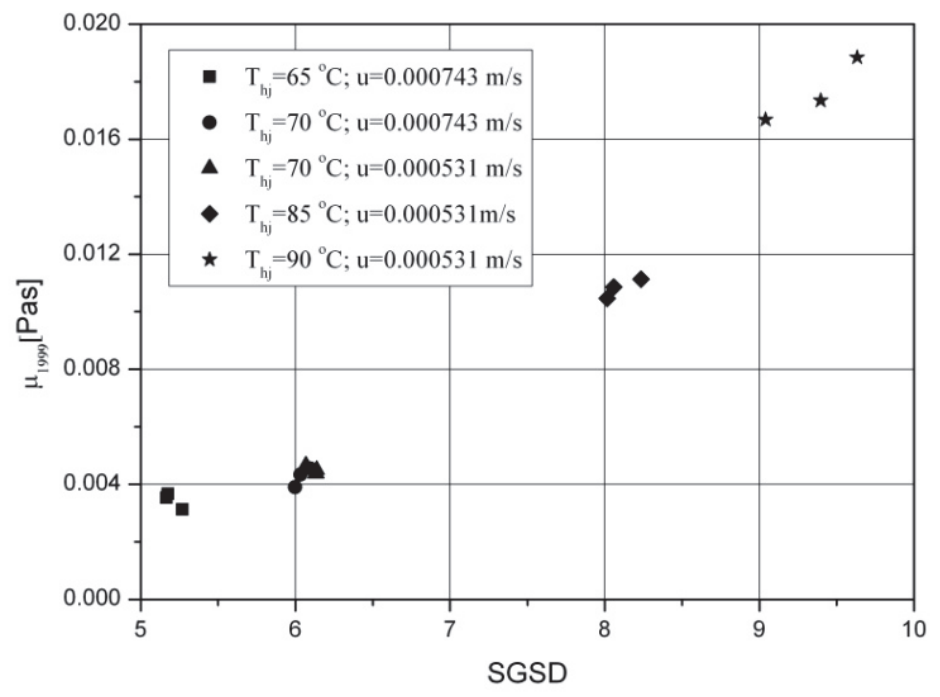

Fig. 7. Dependence of $\mu_{1999}$ on $S G S D$ - $\mu_{1999}$ apparent viscosity of gelatinized starch paste at the maximum shear rate $19991 / \mathrm{s}$ )

In Fig. 8 there are shown the examples how apparent viscosity of gelatinized starch dispersion depends on the value of shear rate. The results of viscosity measurement suggested that gelatinized starch dispersions obtained during our experiments were shear-thinning fluid of thixotropic properties. 


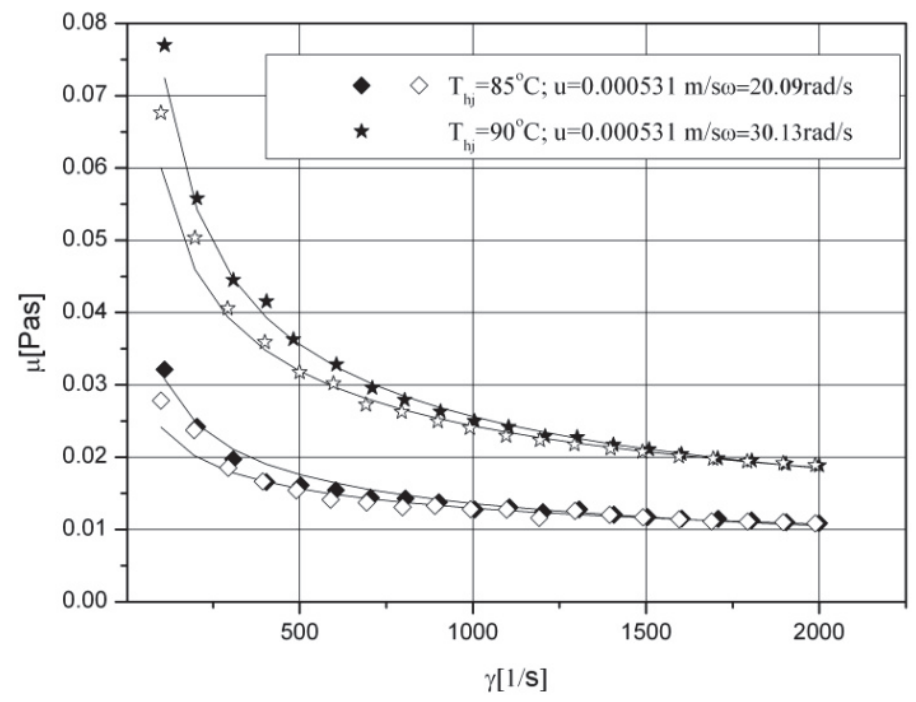

Fig. 8. Apparent viscosity of gelatinized starch as a function of shear rate: black points denote ascending segment of shear cycle, while white points denote descending segment of shear cycle, while lines are the approximation of the results using the power law model

Additionally, the rheological properties of gelatinized starch were approximated using a power law equation. The consistency coefficient $(k)$ and flow behaviour index $(n)$ were calculated for the ascending and descending segments of shear cycle. A relatively good correlation of these parameters with the values of $A B S$ is shown in Figs. 9 and 10.

It confirms the results from our previous work (Hubacz et al. 2016), where a good correlation of the power law parameters with $A B S$ was obtained for starch dispersions after their processing in the CTF apparatus at $T_{h j} \geq 85^{\circ} \mathrm{C}$, but at the different than now values of $u$ and $\omega$. The data in Fig. 10 also confirmed the literature observation (Rao et al., 1997) that the shear-tinning properties of starch dispersion increase with the progress of starch thermal processing.

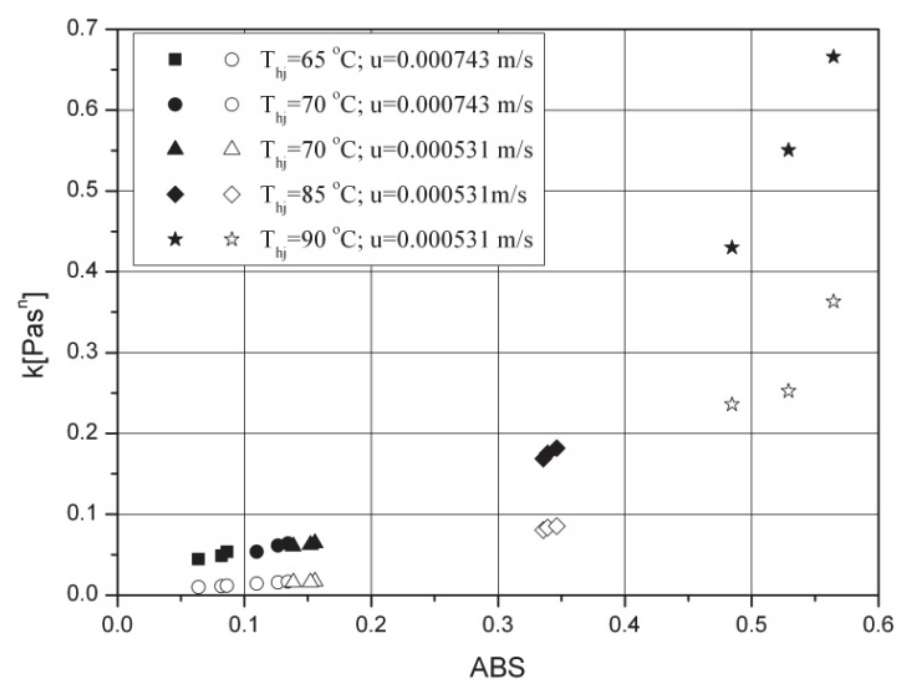

Fig. 9. Consistency coefficient as a function of $A B S$ : black points denote ascending segment of shear cycle, while white points denote descending segment of shear cycle 


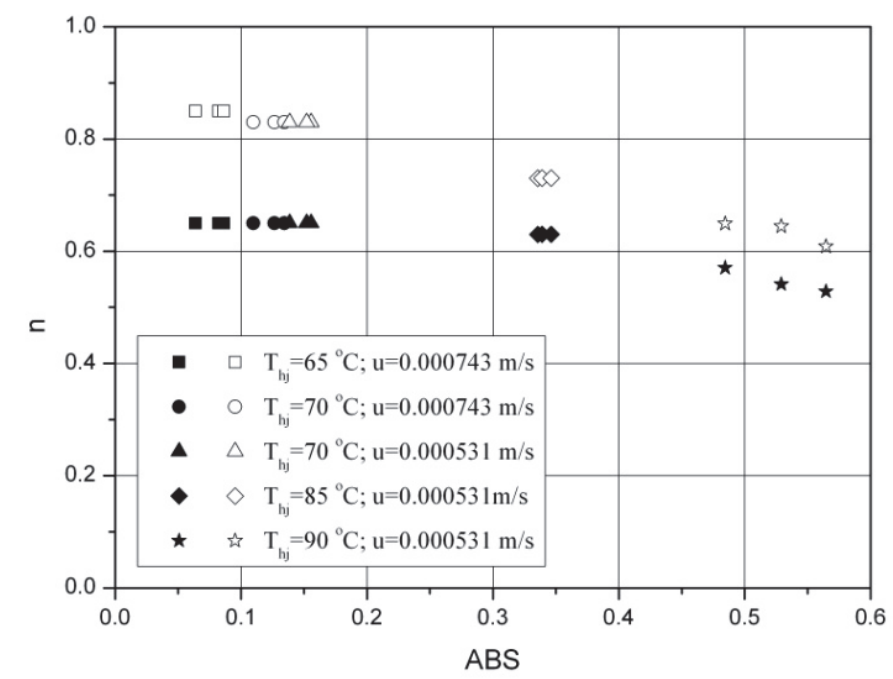

Fig. 10. Flow behaviour index as a function of $A B S$ : black points denote ascending segment of shear cycle, while white points denote descending segment of shear cycle

\subsection{Comparison of CTF apparatuses of different geometries}

The reason for the computer simulation of starch gelatinization in the CTF apparatus investigated in this work (apparatus I) was the comparison with other two CTF apparatuses (apparatus II and apparatus III) investigated by Hubacz et al. (2013). Because, the volumes of apparatuses II and III were bigger than the volume of apparatus I this comparison should provide interesting information concerning scaling-up of the process.

In Table 4, there are shown the values of degree of starch gelatinization (DSG) calculated during numerical simulation of the process in CTF apparatuses at different values of operating parameters. Additionally, the amount of the mechanical energy connected with the rotor rotation was provided in Table 4. In order to compare the results obtained for three different geometries of the apparatus this energy was calculated using the following equation (Hubacz et al., 2013):

$$
E=\frac{\omega R_{1} \iint \tau_{r \theta} d A}{u \pi\left(R_{2}^{2}-R_{1}^{2}\right)}
$$

where $E$ denotes the amount of the mechanical energy (connected with the rotor rotation) needed for the production of a volume unit of the gelatinized starch, $\tau_{r \theta}$ is the angular component of shear stress tensor at the rotor surface calculated from the numerical simulations, $d A$ is the differential surface of the rotor. In order to correctly understand the obtained results it should be also mentioned that the applied model of the process predicted Taylor vortex flow for $\omega \leq 11.56 \mathrm{rad} / \mathrm{s}$ in the case of apparatus II and III (Hubacz et al., 2013). Meanwhile, in the cases of apparatus I the model predicted Taylor vortex flow only for $\omega=19.27 \mathrm{rad} / \mathrm{s}$. This arises from the fact that the CTF apparatus used in this work was the smallest one (see Table 4). According to the results in Table 4 the degree of starch gelatinization obtained in the case of apparatus I and apparatus II were similar. However, the energy needed for the process was significantly bigger in apparatus II. This means that the increase in the apparatus size while keeping the same value of gap width is not really profitable. On the contrary, the significant reduction in the value of $E$ was obtained when apparatus III with the bigger gap width was used. Although most of the values of DSG was smallest in the case of apparatus III, the additional profit of this apparatus could be the occurrence of Taylor vortices at the lower value of $\omega$ than in the case of apparatus I (see the value of DSG for $\omega=11.56 \mathrm{rad} / \mathrm{s}, u=0.00099 \mathrm{~m} / \mathrm{s}$ and $T_{h j}=65^{\circ} \mathrm{C}$ in Table 4). 
Table 4. Results of computer simulation of starch gelatinization in a CTF apparatus

\begin{tabular}{|c|c|c|c|c|c|c|c|c|}
\hline \multirow{2}{*}{$\begin{array}{c}\omega \\
\mathrm{rad} / \mathrm{s}\end{array}$} & \multirow{2}{*}{$\begin{array}{c}u \\
\mathrm{~m} / \mathrm{s}\end{array}$} & \multirow{2}{*}{$\begin{array}{c}T_{h j} \\
{ }^{\circ} \mathrm{C}\end{array}$} & \multicolumn{2}{|c|}{$\begin{array}{c}\text { Apparatus I } \\
R_{1}=0.0125 \mathrm{~m} ; d=0.005 \mathrm{~m}\end{array}$} & \multicolumn{2}{c|}{$\begin{array}{c}\text { Apparatus II* } \\
R_{1}=0.021 \mathrm{~m} ; d=0.005 \mathrm{~m}\end{array}$} & \multicolumn{2}{c|}{$\begin{array}{c}\text { Apparatus III* } \\
\mathrm{R}_{1}=0.016 \mathrm{~m} ; d=0.01 \mathrm{~m}\end{array}$} \\
\cline { 5 - 11 } & & & $\mathrm{DSG}$ & $\mathrm{E}\left[\mathrm{J} / \mathrm{m}^{3}\right]$ & $\mathrm{DSG}$ & $\mathrm{E}\left[\mathrm{J} / \mathrm{m}^{3}\right]$ & $\mathrm{DSG}$ & $\mathrm{E}\left[\mathrm{J} / \mathrm{m}^{3}\right]$ \\
\hline 5.78 & 0.00099 & 65 & 0.55 & 1373.48 & 0.55 & 3007.5 & 0.19 & 640.98 \\
\hline 11.56 & 0.00099 & 65 & 0.56 & 4330.62 & 0.76 & 11543.54 & 0.65 & 3135.3 \\
\hline 19.27 & 0.00099 & 65 & 0.70 & 13615.52 & 0.72 & 42446.3 & 0.66 & 9483.98 \\
\hline 19.27 & 0.00099 & 60 & 0.40 & 13154.69 & 0.46 & 40834.57 & 0.35 & 9028.39 \\
\hline 5.78 & 0.00099 & 85 & 0.99 & 1556.66 & 0.99 & 3392.72 & 0.44 & 675.36 \\
\hline 11.56 & 0.00099 & 85 & 0.99 & 4904.24 & 1 & 11611.91 & 0.99 & 3397.07 \\
\hline 19.27 & 0.00099 & 85 & 1.00 & 14226.22 & 1 & 44845.26 & 0.99 & 10143.86 \\
\hline
\end{tabular}

${ }^{*}$ results from Hubacz et al. (2013)

\section{CONCLUSIONS}

The granule size distribution in gelatinized starch dispersion was more strongly influenced by the processing temperature than by other operating parameters. An increase in the heat jacket temperature caused an increase in the values of $D$ and $S D$. However, the average granule diameter of gelatinized starch also increased when the axial flow was reduced or the rotor rotation velocity increased. The concentration of dissolved starch and starch granule swelling degree depended on the operating parameters in a similar way. However, the changes in $\omega$ or $u$ seemed to have little influence on SGSD when the starch processing was conducted at $T_{h j} \leq 70^{\circ} \mathrm{C}$. This was attributed to different phenomena, which may dominate the process at the lower and at the higher temperature. Nevertheless, the problems with the separation of water, which was not absorbed by starch granules during the processing, from these granule could also affect the value of SGSD especially in the case of the low value of the processing temperature. This can be the second reason why the changes in $\omega$ or $u$ did not affect the value of SGSD significantly at $T_{h j} \leq 70^{\circ} \mathrm{C}$.

The significant increase in the value of $D$ and $A B S$ when $\omega$ was changed from 10.04 to 20.09 could be related to the transition from laminar to Taylor vortex flow pattern inside the apparatus. This conclusion was also supported by numerical simulation of the process.

The gelatinized starch dispersion obtained after treatment in the CTF apparatus consisted of sheartinning fluids of thixotropic properties. Moreover, a good correlation of starch dispersion apparent viscosity with $A B S, S G S D$ or $D$ was observed. Meanwhile, the increase in the value of the apparent viscosity and in shear-tinning behaviour of dispersion was associated with the progress of starch processing in the CTF apparatus.

Finally, simulation results of starch processing in CTF apparatuses indicated that the apparatus scalingup without increasing the width of the gap between cylinders results in higher mechanical energy consumption per unit of processed starch slurry.

\section{SYMBOLS}

ABS light absorbance

$C_{p} \quad$ specific heat capacity, $\mathrm{J} /(\mathrm{kg} \cdot \mathrm{K})$

$D \quad$ average starch granule diameter, $\mu \mathrm{m}$ 
$D_{i} \quad$ starch granule diameter associated with i-range of this diameter values, $\mu \mathrm{m}$

$D_{j} \quad$ diffusion (dispersion) coefficient of species $\mathrm{j}, \mathrm{m}^{2} / \mathrm{s}$

$D S G \quad$ degree of starch gelatinization

$F_{n k}\left(D_{i}\right) \quad$ cumulative distribution function of granule number in kth granule population

$h \quad$ height of the zone in viscometer where a sample was sheared, $\mathrm{mm}$

$k \quad$ consistency coefficient, Pas $^{\mathrm{n}}$

$n \quad$ flow behavior index

$n_{k} \quad$ number of starch granules in kth granule population, respectively

$Q \quad$ volumetric flow rate of the starch slurry measured at the inlet conditions, $\mathrm{m}^{3} / \mathrm{s}$

$r_{1}, r_{2}, r_{3}, r_{4} \quad$ radii of viscometer cylinders, $\mathrm{mm}$

$R_{1}, R_{2} \quad$ radii of rotor and outer cylinder, respectively, $\mathrm{m}$

$S D \quad$ standard deviation of granule size distribution, $\mu \mathrm{m}$

SGSD $\quad$ starch grain swelling degree

$T \quad$ temperature, ${ }^{\circ} \mathrm{C}$

$u \quad$ superficial velocity of starch slurry inside the apparatus, $\mathrm{m} / \mathrm{s}$

$X \quad$ mass fraction

$X_{s 0} \quad$ the total mass fraction of starch in the slurry

$X_{s g} \quad$ the mass fraction of gelatinized starch

Greek symbols

$\delta_{n 1, n 2} \quad$ the biggest difference between the cumulative distribution functions of two compared populations of starch granule diameters

$\mu \quad$ the fluid apparent viscosity, Pas

$\omega \quad$ rotor angular velocity, $\mathrm{rad} / \mathrm{s}$

$\begin{array}{ll}\text { Subscripts } & \\ h j & \text { heat jacket } \\ i & \text { range of granule diameter values } \\ j & \text { species } \\ k & \text { population of starch granules } \\ s g & \text { starch gelatinized } \\ \text { s0 or } 0 & \text { starch before gelatinization }\end{array}$

\section{REFERENCES}

Baks T., Ngene I.S., Van Soest J.J.G., Janssen A.E.M., Boom R.M., 2007. Comparison of methods to determine the degree of gelatinisation for both high and low starch concentrations. Carbohydr. Polym., 67, 481-490. DOI: 10.1016/j.carbpol.2006.06.016.

Brandam C., Meyer X. M., Proth J., Strehaiano P., Pinguad H., 2003. A original kinetic model for the enzymatic hydrolysis of starch during mashing. Biochem. Eng. J., 13, 43-52. DOI: 10.1016/S1369-703X(02)00100-6.

Dłuska E., Markowska-Radomska A., 2010. Regimes of multiple mmulsions of W-1/O/W-2 and O-1/W/O-2 type in the continuous Couette-Taylor flow contactor. Chem. Eng. Technol., 33, 113-120. DOI: 10.1002/ceat.200900278.

Dłuska E., Hubacz R., 2000. Mass transfer in the two-phase helicoidal contactor. Inż. Chem. Proces., 21, 1, 103113.

Hellwig Z., 1998. Elementy rachunku prawdopodobieństwa i statystyki matematycznej. $13^{\text {th }}$ edition, WNT, Warszawa.

Hubacz R., Buczyńska M., 2011. Starch gelatinisation in Couette-Taylor flow apparatus. Chem. Process Eng., 32, 267-279. DOI: 10.2478/v10176-011-0021-7.

Hubacz R., Ohmura N., Dluska E., 2013. Intensification of starch processing using apparatus with Couette-Taylor flow. J. Food Process Eng., 36, 6, 774-785. DOI: 10.1111/jfpe.12046. 
Hubacz R., Masuda H., Horie T., Ohmura N., 2016. Starch granules swelling during gelatinization in CouetteTaylor flow apparatus. Proceedings of the 22nd of Chemical and Process Engineering. Spała, Poland, 5-9 September 2016, 435-444.

Lagarrigue S., Alvarez G., Cuvelier G., Flick D., 2008. Swelling kinetics of waxy maize and maize starches at high temperatures and heating rates. Carbohydr. Polym., 73, 148-155. DOI: 10.1016/j.carbpol.2007.11.018.

Malumba P., Jacquet N., Delimme G., Lefebvre F., Bera F., 2013. The swelling behaviour of wheat starch granules during isothermal and non-isothermal treatments. J. Food Eng., 114, 199-206. DOI: 10.1016/j.jfoodeng.2012.08.010.

Mayra Q.-P., Kim W.-S., 2015. Agglomeration of Ni-Rich hydroxide in reaction crystallization: Effect of Taylor vortex dimension and intensity. Cryst. Growth Des., 15, 1726-1734. DOI: $10.1021 / \mathrm{cg} 501727 \mathrm{v}$.

Masuda H., Horie T., Hubacz R., Ohmura N., 2013. Process intensification pf continuous starch hydrolysis with a Taylor-Couette flow reactor. Chem. Eng. Res. Des., 91,11, 2259-2264. DOI: 10.1016/j.cherd.2013.08.026.

Patel B. K., Seetharaman K., 2006. Effect of heating rate on starch granule morphology and size. Carbohydr. Polym., 65, 381-385. DOI: 10.1016/j.carbpol.2006.01.028.

Ramezani M., Kong B., Gao X., Olsen M.G., Vigil R.D., 2015. Experimental measurement of oxygen mass transfer and bubble size distribution in and air-water multiphase Taylor-Couette vortex bioreactor. Chem. Eng. J., 279, 286-296. DOI: 10.1016/j.cej.2015.05.007.

Rao M.A., Okechukwu P.E., Da Silva P.M.S., Oliveira J.C., 1997. Rheological behaviour of heated starch dispersions in excess water: role of starch granule. Carbohydr. Polym., 33, 273-283. DOI: 10.1016/S01448617(97)00025-8.

Sakonidou E.P., Karapantsios T.D., Raphaelides S.N., 2003. Mass transfer limitations during starch gelatinization. Carbohydr. Polym., 53, 53-61. DOI: 10.1016/S0144-8617(03)00010-9.

Singh N., Singh S., Isono, N., Noda T., Singh A. M., 2009. Diversity in amylopectin structure, thermal and pasting properties of starches from wheat varieties/lines. Int. J. Bio Macromol. 45, 298-304. DOI: 10.1016/j.ijbiomac.2009.06.005. 